\title{
The Research on the Load Prediction for Ice Storage System Based on Rough Set
}

\author{
Shi XiaoXia and Wang Dong \\ Automation Department, Beijing University of Civil Engineering and Architecture, \\ Beijing, China, 100044 \\ shixiaoxia@bucea.edu.cn
}

\begin{abstract}
The accurate cooling load prediction of ice storage system is the basis and foundation for optimization control. In order to implement the optimal control of ice storage system, the accurate cooling load prediction is very important. This paper proposes that the SVM (support vector machine) combined with rough set is used for the load prediction of ice storage system. The simulation results proved that it opens a new avenue in load forecasting. SVM based on RS method is a promising alternative approach for cooling load prediction in a building.
\end{abstract}

Keywords: ice storage system, load prediction, SVM, rough set

\section{Introduction}

Buildings are responsible for $73 \%$ of the total electricity consumption. Heating, ventilation, and air conditioning (HVAC) systems generate $33 \%$ of the total building energy consumption $[1,2]$. Therefore, it is both economically and environmentally significant to reduce HVAC energy consumption.

Along with the fast development of modern construction in China, the energy consumption of air conditioning increases rapidly and is fast approaching the international level. As the environmental temperature changes, the cooling load and the electricity consumption change correspondingly. During the periods of high outdoor temperatures, they are much higher than those during the periods with lower outdoor temperatures. To meet the peak electricity demand, utilities are forced to introduce new power generating unit, which is termed as peak regulating unit. It is well known that ice storage technology is regarded as a feasible approach for peak-load shifting in the cooling season, as the price of electricity during off-peak hours is much lower than during peak hours [3]. Although ice storage processes consume more power than conventional processes with the same cooling capacity, the operating costs of ice storage processes are much lower, and their applications in buildings have been on the rise in recent years [4-6]. How to control the operating mode of the ice storage system to achieve energy saving? In fact, a well-controlled ice storage system should meet the following two requirements: one is that an ice storage volume must meet the requirement of air conditioning cooling in the next day. The other is that an ice storage system should be scheduled to release cold, and the ice in the storage tank is neither exhausted ahead of schedule nor remained at the end of schedule. Therefore, an accurate prediction of the building load is the basis of effective implementation of an ice storage system. This paper proposes a method which uses SVM combined with RS theory to predict the load of the ice-storage system. It provides a new perspective for the researchers on the load prediction of the ice storage system.

\subsection{Ice Storage System}


The ice storage systems use the latent heat of ice to store cooling energy. Ice storage system stores energy by producing ice during off-peak periods and releases the stored energy during the peak periods. It is an effective measure for shifting peak period power demand to the off-peak nighttime hours. Since the electricity price during off-peak hours is lower than that in peak hours, applying ice storage air conditioning system is a costeffective measure, and its application is increasing [7,8].

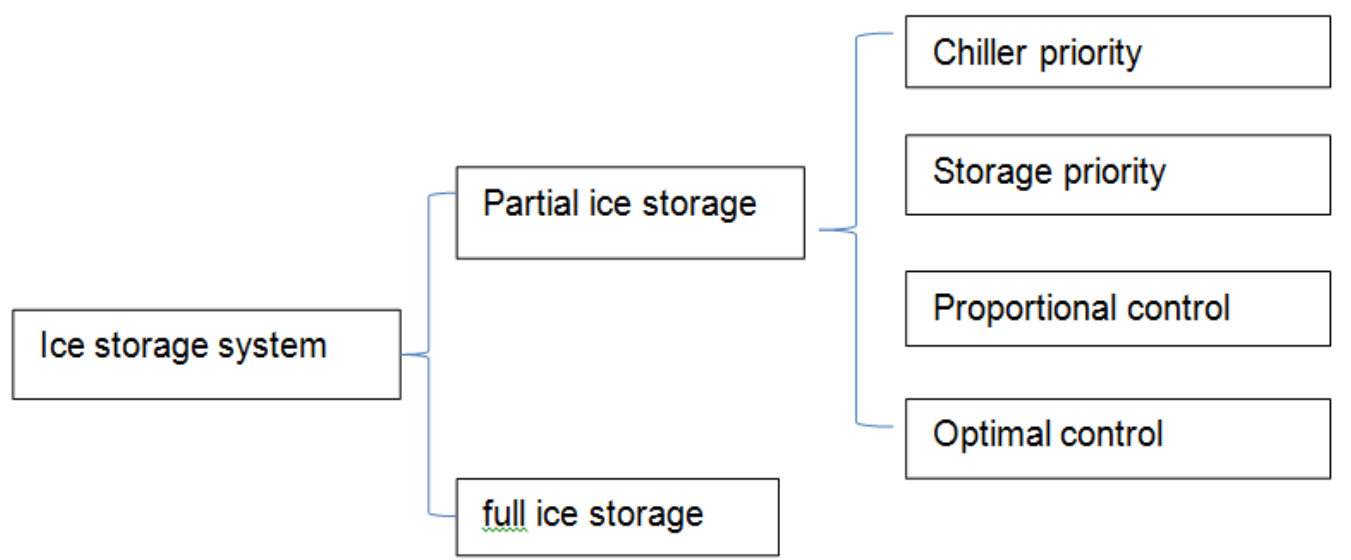

Figure 1. The Usual Control Strategy for Ice Storage System

In ice storage systems, ice can be made (i.e., ice tank is charged) when the cost of electricity is lower; the ice tank can supply cooling to the building (i.e., the ice tank is discharged) during peak hours, when the cost of electricity is higher [9]. The stored cooling energy is used to meet the air conditioning requirements during the peak periods. Thus, part of the power demand is shifted from peak daytime periods to off-peak night time periods [3]. According to the load characteristics and the cost of the electricity, the operating strategies of ice storage system can be classified as full ice storage and partial ice storage. In partial ice storage systems, the conventional air conditioning system operates to meet part of the peak period cooling load, and the rest is met by drawing from storage. Four control strategies were investigated for partial ice storage systems: optimal control and three conventional controls (chiller priority, constant proportion and storage priority control), just shown in Figure 1.

For all three conventional strategies, the off-peak control rule is to recharge the storage as quickly as possible to be prepared for the next on-peak period. During on-peak periods, however, the strategies differ. Chiller-priority control meets all of those cooling loads for which the chiller capacity is sufficient. Loads that exceed the chiller capacity, are met in parallel by the chiller and the ice storage. Consequently, chillers that are supposed to operate in conjunction with an ice storage system under chiller-priority control must be downsized to make the ice storage system feasible. Typical values of the chiller capacity are about $80 \%$ of the peak cooling load $[10,11]$.

Constant proportion control prescribes that at each on-peak hour a constant load fraction of the current cooling load is met by the ice storage. A load fraction value of $25 \%$ was found to provide good load-shifting performance without premature storage depletion for a wide spectrum of cases. The chiller is fully sized, i.e., sized to meet the peak load directly.

Storage priority is very similar to constant proportion control except that the load fraction is not constant for a whole month or season, but computed daily anew from cooling load predictions (over the next on-peak period) such that the storage is fully depleted at the end of the next on-peak period. This strategy ensures that the available storage is always fully utilized. 
In accordance with its definition, storage priority completely discharges the entire storage over the on-peak periods. These three conventional controls keep the tank full over the entire weekend in preparation for the next on-peak period.

All in all, chiller priority cannot develop the ability of load shifting. Storage priority not only makes the ice in exhaustion in advance, but also makes the chillers work in the state of low load for a long time, which result in the unreasonable operation.

Optimal control in the given context is defined as the control trajectory that minimizes the combined energy and demand charge of the electrical utility bill for cooling related and non-cooling related electricity use. During the week-days, optimal control behaves similarly to storage priority. The most significant difference is that optimal control recharges the empty tank more slowly using the entire off-peak period. Over the weekend, optimal control goes through some irregular charging and discharging cycles but is completely recharged at the onset of the first on-peak period of the week.

It is well known that the ice storage system is not an energy saving system, but balances the city power load through the load transfer function. The users can save the costs by the full use of the local difference of the peak and valley price. Table1 gives the commercial electricity prices during the different time in Beijing.

Table 1. The Commercial Electricity Prices in Beijing (Unit: Yuan/KWh)

\begin{tabular}{c|c|c|c}
\hline \hline $\begin{array}{c}\text { On-peak } \\
\text { price }\end{array}$ & $\begin{array}{c}\text { Peak } \\
\text { price }\end{array}$ & Flat price & Valley price \\
\hline 1.5065 & 1.378 & 0.8595 & 0.3658 \\
\hline $11: 00-$ & $10: 00-$ & $7: 00-$ & $23: 00-7: 00$ \\
$13: 00$ & $11: 00$ & $10: 00$ & \\
$20: 00-$ & $13: 00-$ & $15: 00-$ & \\
$21: 00$ & $15: 00$ & $18: 00$ & \\
& $18: 00-$ & $21: 00-$ & \\
& $20: 00$ & $23: 00$ & \\
& & & \\
\hline \hline
\end{tabular}

We can see from Table 1, if we can make full use of the valley price, which not only relieve the pressure of the city electricity, but also save energy and costs. It is the decisive factor for daily operation cost to determine how to control the ice storage amount during the valley price time reasonably, as well as the ice melting speed in the peak period and flat price time. Through the exploration and research for many years, it is generally believed that the effective way to solve the control problem is the optimal control based on the air conditioning load prediction.

\subsection{The Method of Load Prediction}

Accurate prediction of the dynamic air-conditioning load in a building is a key for the optimal control of a HVAC system. Many techniques have been proposed in the last few decades for short-term load forecasting. The traditional techniques include autogressive integrated moving-average (ARIMA) model, linear regression (LR) technique, neuralnetwork (ANN) model, and grey model [3]. Kalogirou, et al., [12] used back propagation neural networks to predict the required heating load of buildings. Minoru Kawashima et al. [13] described an artificial neural network model to predict the next day's total cooling load. Kreider and Wang [14] demonstrated an automated load prediction using the ANN model. Zhang Lin [15] proposed an improved Support Vector Machines algorithm based on time sequence to apply in load forecasting. Li Qiong, et al., [16] apply radial basis function (RBF) to predict the load of a building in GuangZhou. Chen Liu [17] proposed a model of wavelet neural network for predicting the air-conditioning load. An SVM model and back-propagation (BP) neural network model are both used for the hourly air 
conditioning load prediction of an office building in summer months in Guangzhou area by Li Qiong, et al., [18], and the simulation results show that the SVM model more accuracy and more effective than BP neural network. MacArthur, et al., [19] and Spethmann [20] developed a prediction method based on the autoregressive integrated moving average (ARIMA) model and applied it to an optimal cold storage controller. Li Xuemei, et al., [21] proposed an optimal model which is based on stimulated annealing particle swarm optimization algorithm that combines the advantages of PSO algorithm and SA algorithm. In Ref [21], it illuminated that because of its strong non-linear mapping ability, artificial neural networks (ANN) are widely accepted as a technology offering an alternative way to tackle complex and ill-defined problems, which have been popularly applied to predict the building cooling load and building energy consumption. ANNs-based models seem to obtain improved and acceptable performance in cooling load forecasting issue, however, the conventional ANNs still suffer from several weaknesses such as the need for a large number of controlling parameters, the difficulty in obtaining stable solutions, the danger of over fitting and thus the lack of generalization capability. However, the time series method often considers only short-term load prediction, and it will have large deviation when considering the long-term load prediction [22].

As we all know that accurately predicting the building cooling load is a challenging work. Cooling load in the building is affected by many factors. For an accurate forecasting model, it is important to understand which factors influence the load level most. Such knowledge is often acquired from experienced operators. However, manual selection of factors pertinent to prediction task would not guarantee an optimal solution: the inaccurate estimations result in inaccurate predictions. Hence, there is a need for a reliable model that can select relevant factors automatically from historical data. Rough set (RS) theory, which can be used for attribute reduction, provides a solvable method for this problem. By attribute reduction, irrelevant factors to the tasks can be identified and removed. RS has proved to be very effective in many practical applications. However, in RS theory, the deterministic mechanism for the description of error is very simple. Therefore, the rules generated by RS are often unstable and have low classification accuracies. So RS cannot predict loads with high accuracy. RS theory provides useful techniques to reduce irrelevant and redundant attributes from a large database with a lot of attributes.

In recent years, support vector machine (SVM), developed by Vapnik and his coworkers in 1995, and has been widely used in classification forecasting and regression [4]. In this paper, based on the RS theory for the attribute reduction, we try to introduce the theory of SVM into the forecasting of hourly building cooling load. The SVM theory based on RS theory establishes a building cooling load prediction model. The steps are proposed. Figure 2 shows that firstly, some reductions can be derived from crude data based on RS theory and relevant factors can automatically be obtained from historical data. Then SVM is trained to learn in order to predict the load. The simulation results for a building in Beijing show that SVM combined with RS theory is feasible.

\section{The RS Theory}

The RS theory is one of the mathematical tools that can tackle uncertain and fuzzy data. It is proposed by Pawlak $\mathrm{z}$ in 1982. The RS theory is one of the promising data mining techniques. The theory was characterized with the attributes reduction and association rules extraction ability. Compared with other tools, the most important advantage of Rough Set is that it does not require any pre-knowledge about the data. The main object of Rough Set is to extract rules from Information System (IS). An IS can be demonstrated as $I=\langle U, A\rangle$, where $\mathrm{U}$ denotes a collection of limited and nonempty objects $U=\left\{x_{1}, x_{2}, \ldots, x_{n}\right\}$ and $\mathrm{A}$ denotes a collection of nonempty and limited attributes 
as $A=\left\{a_{1}, a_{2}, \ldots a_{m}\right\}$. For each $a_{i} \in A, a_{i}: U \rightarrow V a_{i}$ where $V a_{i}$ denotes the value field of attribute ai, A can further be divided into two collections that are conditional attribute collection C and Decision attribute collection D with no intersection. The kind of IS are called Decision Information System (DIS) or Decision Table (DT) [23].

\section{SVM}

Support Vector Machines (SVMs), derived from statistical learning theory and VCdimension theory, have been widely used in many fields and show good performance (Vapnik, 1998). Good generalization ability is an important characteristic of SVMS.

SVM is a novel type of learning machine, gaining popularity due to its many attractive features and promising empirical performance. SVM has been successfully employed to solve nonlinear regression and time series problems. The main advantage of SVM is that it adopts the structure risk minimization (SRM) principle, which has been shown to be superior to the traditional empirical risk minimization (ERM) principle, employed by conventional neural networks. SRM seeks to minimize an upper bound of the generalization error consisting of the sum of the training error and a confidence level based on VC dimension, which is different from commonly used ERM principle that only minimizes the training error. This method has been proven to be very effective for addressing general purpose classification and regression problems [17]. The basic idea of SVM for regression is to introduce kernel function, map the input space into a highdimensional feature space via a nonlinear mapping and to perform a linear regression in this feature space $[24,25]$.

\section{A Building Cooling Load Prediction Model}

\subsection{The Steps for Load Forecasting Model}

In order to predict the cooling load for ice-storage system with the method of SVM based on RS theory, the steps are as follows:

Step 1: Attribute reduction using RS theory from historical data. The following is the implementation:

(1) To construct the corresponding vector sets according to the preprocessed data.

(2) To reduce attribute using RS theory for each vector set.

(3) To store the dimension-reduced vector.

(4) To extract the features and find the decision factors.

(5) To take data discretization with equidistant discretization method.

Step 2: To take the discretized data as the inputs and forecast the load with SVM. The flow chart is shown with Figure 2. 


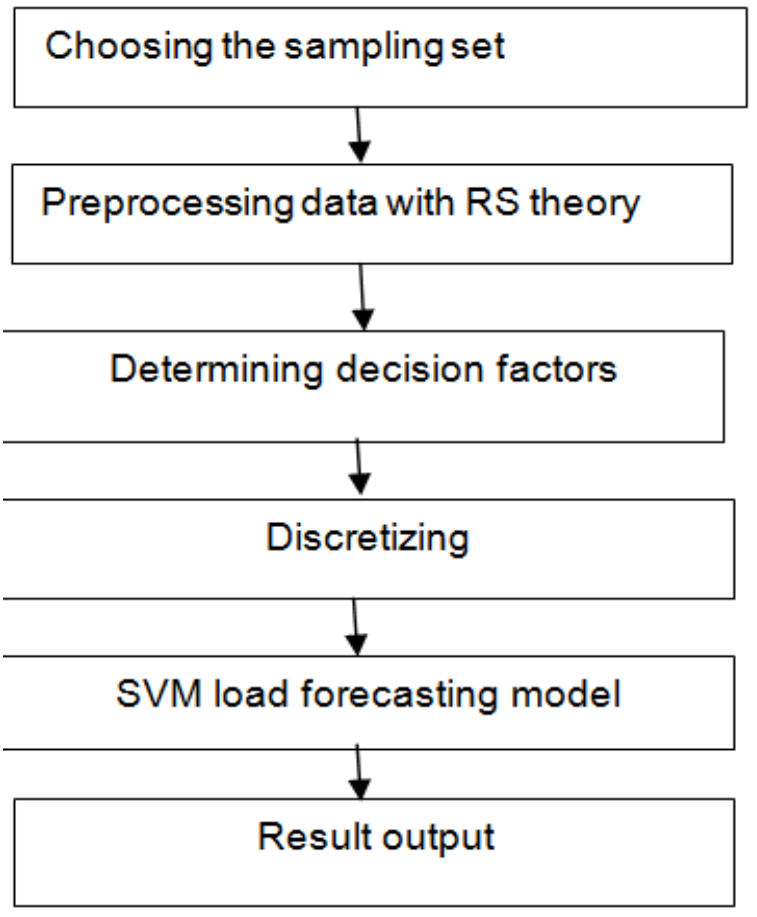

Figure 2. The Flow Chart of the Algorithm

\subsection{The Model based on the Combination of RS and SVM}

The common advantage of RS and SVM is that they do not need any additional information about data like probability in statistics or grade of membership in fuzzy-set theory. RS has proved to be very effective in many practical applications. RS theory provides useful techniques to reduce irrelevant and redundant attributes from a large database with a lot of attributes. SVM has the ability to approach any complex functions and possess as a good robustness to noise.

To predict the load for the ice storage air-conditioning system the ideal input samples will be the following parameters: the outdoor dry bulb temperature, outdoor wet bulb temperature or dew point temperature, atmospheric pressure, cloud state, cloud cover (010 levels), relative humidity (absolute temperature), outdoor air enthalpy, outdoor air density, wind speed, wind direction, the sun direct radiation intensity, diffuse solar radiation intensity, the ground scattered radiation intensity, rainfall, snowfall, indoor load, the indoor equipment heat load. But it is too complex to model load predicting model with so many input parameters. So at first we use the RS theory to find the decision factor for the load predicting. We can get six input parameters for the building cooling prediction model: Tt, Tt-1, Tt-2 denote the outdoor dry bulb temperature $(\circ \mathrm{C})$ at the time $\mathrm{t}, \mathrm{t}-1, \mathrm{t}-2$, respectively, Dt is the relative humidity (\%) and Lt and Lt-1are the solar radiation intensity (W/m2) at time $t$ and $\mathrm{t}-1$ respectively. After determining the input parameters, the cooling load as the output, we can establish the load forecasting model with SVM. Suppose that all the normalized input parameters compose a vector Xi ( $\mathrm{i}$ represents one input sample), and $\mathrm{Yi}$ is the normalized building cooling load under the input sample $\mathrm{i}$. when the total number of samples is $\mathrm{N}$, the sample set is defined as $\left\{\left(\begin{array}{ll}X_{i} & Y_{i}\end{array}\right)\right\}_{i=1}^{N}$. Therefore, SVM approximates the relationship between the output and input parameters using the following form:

$$
Y=f(X)=W \cdot \phi(X)+b
$$


Where $X_{i}=\left\{\begin{array}{llllll}T_{i} & T_{i-1} & T_{i-2} & D_{i} & L_{i} & L_{i-1}\end{array}\right\} \cdot \phi(X)$ represents the high dimensional feature space which is nonlinearly mapped from the input space $X$, coefficients $W$ and $b$ are estimated by minimizing the regularized risk function shown

$$
\begin{aligned}
& \min Q=\frac{1}{2}\|W\|^{2}+c \sum_{i=1}^{N}\left(\xi_{i}+\xi_{i}^{*}\right) \\
& \text { s.t. }\left\{\begin{array}{l}
Y_{i}-f\left(X_{i}\right) \leq \varepsilon+\xi_{i} \\
f\left(X_{i}\right)-Y_{i} \leq \varepsilon+\xi_{i}^{*} \quad(i=1,2, \cdots, N) \\
\xi_{i}^{*}, \xi_{i} \geq 0
\end{array}\right.
\end{aligned}
$$

In eq.(2), the first term $\|W\|^{2}$ is called regularized term. Minimizing $\|W\|^{2}$ will make the function as flat as possible, thus playing role of controlling the function capacity. The second term is the empirical error measured by $\varepsilon$-insensitive loss function. $C$ is the penalty parameter, which is regularized constant to determine the trade-off between complex function regression model and the accuracy of sample fitting. $\varepsilon$ is the largest error that regression allowed, econtrols the number of the support vectors and the generalization ability, the bigger it is , the less the number of support vectors are. $\xi_{i}$ and $\xi_{i}^{*}$ are positive slack variables respectively.

According to the Lagrange optimization method and duality principle, the optimization problem Eq.(2) can be rewritten as follows:

$$
\begin{aligned}
& \max J=-\frac{1}{2} \sum_{i, j=1}^{N}\left(\alpha_{i}-\alpha_{i}^{*}\right)\left(\alpha_{j}-\alpha_{j}^{*}\right) K\left(x_{i}, x_{j}\right)-\sum_{i=1}^{N} \alpha_{i}\left(y_{i}+\varepsilon\right)+\sum_{i=1}^{N} \alpha_{i}^{*}\left(y_{i}-\varepsilon\right) \\
& \text { s.t. }\left\{\begin{array}{l}
\sum_{i=1}^{N}\left(\alpha_{i}-\alpha_{i}^{*}\right)=0 \\
0 \leq \alpha_{i}, \alpha_{i}^{*} \leq C
\end{array}\right.
\end{aligned}
$$

Here, $W=\sum_{i=1}^{N}\left(\alpha_{i}-\alpha_{i}^{*}\right) \varphi\left(x_{i}\right)$.

In order to improve the calculation efficiency, and prevent individual data from overflowing during the calculation, input and output parameters should be normalized as follows:

$$
Y=f(X)=\sum_{i=1}^{N}\left(\alpha_{i}-\alpha_{i}^{*}\right) K\left(X_{i}, X\right)+b
$$

Where $\alpha_{i}$ and $\alpha_{i}^{*}$ are Lagrange multipliers, $\mathrm{i}$ and $\mathrm{j}$ represent different samples respectively. $K\left(X_{i}, X\right)$ is kernel function. Any function $\mathrm{K}(\mathrm{Xi}, \mathrm{Xj})$ satisfying Mercer's condition can be used as the kernel function [26]. The typical kernel functions include linear function, polynomial function, Gaussian function, and Sigmoid function, etc. among these functions, the Gaussian function can map the sample set from the input space into a highdimensional feature space effectively, which is good for representing the complex nonlinear relationship between the output and input samples. Moreover, there is only one variable needing to be determined, thus, Gaussian function is used widely. Gaussian function is also selected as the kernel function in this paper, whose expression is shown as follows:

$$
\mathrm{K}\left(\mathrm{X}_{\mathrm{i}}, X_{j}\right)=\exp \left(-\left\|X_{i}-X_{j}\right\|^{2} / \sigma^{2}\right)
$$

Where $\sigma 2$ is the width parameter of Gaussian kernel.

The SVM load forecasting model is as Figure 3. 


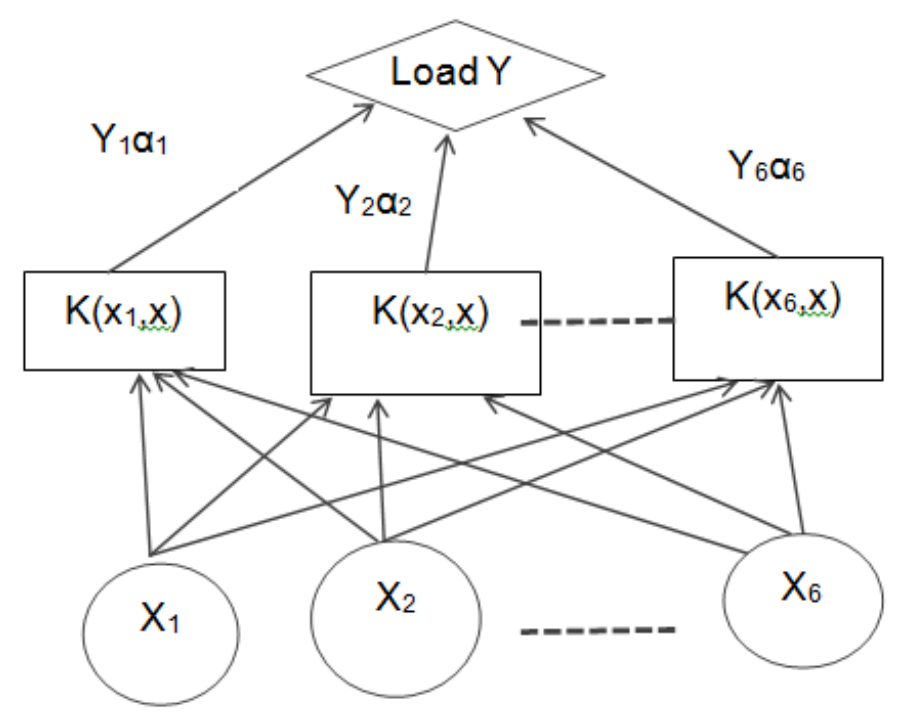

Figure 3. The Scheme of SVM for Load Prediction

\subsection{Application}

An office building located in Beijing, China is selected randomly to justify the feasibility of SVM based on RS theory to establish the building cooling load prediction model. The total building area is $14,957 \mathrm{~m} 2$, where the ground area is $8839 \mathrm{~m} 2$, the underground area is $6118 \mathrm{~m} 2$. DeST is used to calculate the office building's hourly cooling loads, which are taken as the basic values to compare with the predicted values from SVM models. Through repeated experiments, we set $\sigma^{2}=5, C=10, \varepsilon=0.034$, which can be the best for the load forecasting. We choose the data from 1st of June to 9th of June as the training sample. The comparison of the predicting load with SVM based on RS and the actual load is shown in Figure 4, The predicting error is shown in Figure 5. The result shows that the new method can predict the cooling load effectively and accurately.

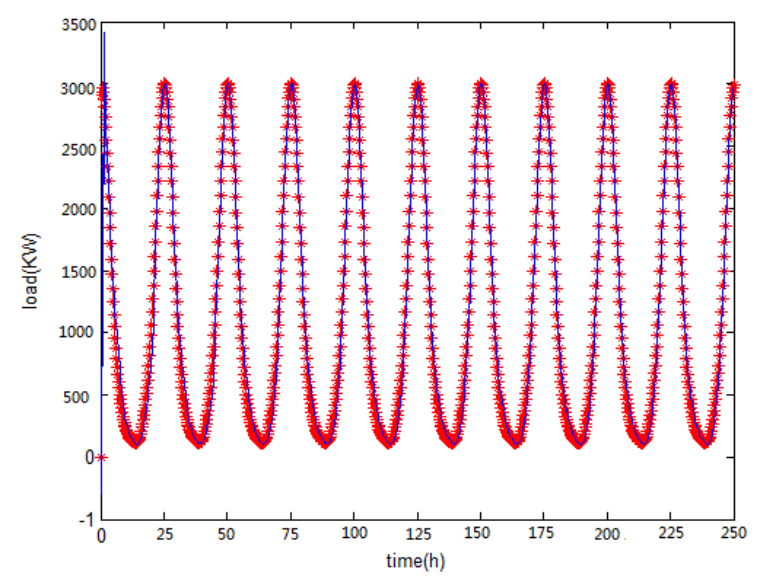

Figure 4. Comparison of the Forecasting with SVM based on RS and Actual Load Curves 


\section{Figure 5. Output Error}

\section{Conclusion}

RS can remove redundant attributes without any classification information loss. SVM is a novel type of learning machine, gaining popularity due to its many attractive features and promising empirical performance.

A novel method of combined RS with SVM models was applied to forecast cooling load of the ice storage air conditioning system. The basic steps are produced. It is a guiding role for the load forecasting. The SVM combined with RS method's quick and correct learning performance makes it open a new avenue in the load forecasting. SVM based on RS method is a promising alternative approach for cooling load prediction in a building.

\section{ACKNOWLEDGEMENTS}

This is supported by the surface project foundation of Beijing Education Commission (051100615).

\section{REFERENCES}

[1] U. S. Doe, "Buildings Energy Data Book", (2009), http://buildingsdatabook.eren.doe.gov/(accessed 11.25.12).

[2] M. Avci, M. Erkoc, A. Rahmani and S. Asfour, "Model predictive HVAC load control in buildings using real-time electricity pricing", Exergy and Buildings, pp. 199-209.

[3] K. Liu, H. Guven, A. Beyee and P. Lowery, "A comparison of the field performance of thermal energy storage (TES) and conventional chller systems", Energy, vol. 19, (1994), pp. 889-900.

[4] J. Pu, G. L. Liu and X. Feng, "Cumulative energy analysis if ice thermal storage air conditioning system”, Applied Energy, vol. 93, (2012), pp. 564-569.

[5] W. S. Lee, Y. T. Chen and T. H. Wu, "Optimization for ice-storage air-conditioning system using particle swarm algorithm”, Applied Energy, vol. 86, (2009), pp. 158-1595.

[6] H. J. Chen, D. W. P Wang and S. L. Chen, "Optimization of an ice-storage air conditioning system using dynamic programming method", Applied Thermal Engineering, vol. 25, (2005), pp. 461-472.

[7] S. M. Hasnain, "Review on sustainable thermal energy storage technology, part II: cool thermal storage", Energy Convers Manage, vol. 39, (1998), pp. 1139-1153.

[8] C. D. Ho, T. Jw, T. L. Hsien, H. Chang and T. C. Chen, "Heat transfer enhancement in cool-thermal discharge systems from ice meltingwith time-velocity variations", Intcommn Heat Mass transfer, vol. 37, (2010), pp. 815-821.

[9] A. Arteconi, N. J. Hewitt and F. Polonara, "State of the art of thermal storage for demand-side management", Apply Energy, vol. 93, (2012), pp. 371-389.

[10] G. P. Henza, M. Krarti and M. J. Brandemuehl, "Guidelines for improved performance of ice storage systems", Energy and Buildings, vol. 35, (2003), pp. 111-127.

[11] J. Pu, G. Liu and X. Feng, "Cumulative exergy analysis of ice thermal storage air conditioning system", Applied Energy, vol. 93, (2012), pp. 564-569.

[12] S. A. Kalogirou, C. C. Neocleous and C. N. Schizas, "Building heating load estimation using artificial neural networks", Proceedings of the 17th international conference on parallel architectures and compilation techniques, (1997). 
[13] M. Kawashima and C. E. Dorgan, "Optimizing system control with load prediction by neural networks for an ice-storage system", ASHRAE Trans, Part 1, vol. 102, (1996), pp. 1169-1178.

[14] J. F. Kreider and X. A. Wang, "Improved artificial networks for commercial building energy use prediction", Proceedings of the ASME Annual Solar Engineering Meeting, Maui, HI, (1992), pp. 361366.

[15] Z. Lin, "Application of support vector machines based on time sequence in power system load forecasting”, Power System Technology, vol. 28, no. 19, (2004), pp. 38-41.

[16] L. Qiong and M. Q. Lin, "Prediction model of hourly air conditioning load of building based on RBF neural network", Journal of South China University of Technology (Natual Science Edition), vol. 36, no. 10, (2008), pp. 25-31.

[17] C. Liu, "Application of wavelet neural network for air-conditioning load prediction", Building Science, vol. 25, no. 10, (2009), pp. 70-73.

[18] L. Qiong, M. Qinglin, H. Yoshino and A. Mochido, "Building air conditioning load prediction model based on support vector machine", HV\&AC, vol. 38, no. 1, (2008), pp. 14-18.

[19] J. W. MacArthur, A. Mathur and J. Zhao, "On-line recursive estimation for load profile prediction", ASHRAE Trans, Part 1, vol. 95, (1989), pp. 621-628.

[20] D. H. Spethmann, "Optimal control for cool storage", ASHRAE Trans. Part 1, vol. 95, (1989), pp. 710721.

[21] L. Xuemei, D. Lixing and L. Lanlan, "A novel building cooling load prediction based on SVR and SAPSO", International Symposium on Computer, Communication, Control and Automation (2010), pp. 528-533.

[22] Z. Junhua, C. Yimin, S. Xiaoxia and W. Dong, "Building cooling load prediction based on time series method and neural networks", The 8th Cross-Strait conference on information science and technology, (2014), pp. 197-204.

[23] J. Li, G. Xia and X. Shi, "Association rules mining from time series based on rough set", Intelligent Systems Design and Applications, ISDA '06, (2006), pp. 509-516.

[24] Q. Li, Q. Meng, J. Cai, H. Yoshino and A. Mochida, "Applying support vector machine to predict hourly cooling load in the building", Applied Energy, vol. 86, (2009), pp. 2249-2256.

[25] J. Mercer, "Function of positive and negative type and their connection with the theory of integral equations", Philos Trans Roy Soc Lond Series A1909, vol. 209, pp. 415-416.

[26] D. Hui, F. He-lin and L. Wu-Ming, "Support vector machines for time series regression and prediction", Journal of system simulation, vol. 18, no. 7, (2006), pp. 1785-1788.

\section{Authors}

Shi XiaoXia, (1976), doctor, a lecturer of BEIJING University of Civil Engineering and Architecture. Researching on system identification and modeling, intelligent control. Load prediction and Energy-saving of the air conditioning system.

Wang Dong, (1989), a student for master degree in automatic control department of BEIJING University of Civil Engineering and Architecture. Research field: Intelligent Building Technology 